Note from the field

\title{
Microwave-assisted hydrothermal extraction of natural malic acid for the synthesis of low transition temperature mixtures
}

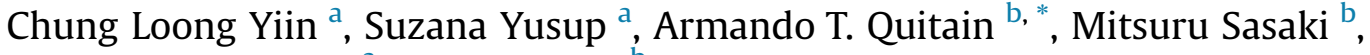 \\ Yoshimitsu Uemura a, Tetsuya Kida ${ }^{\text {b }}$ \\ a Biomass Processing Technology Cluster, Center of Biofuel and Biochemical Research, Mission Oriented Research (Green Technology), Chemical Engineering \\ Department, Universiti Teknologi PETRONAS, Bandar Seri Iskandar, 32610 Tronoh, Perak, Malaysia \\ ${ }^{\mathrm{b}}$ Department of Applied Chemistry and Biochemistry, Kumamoto University, 2-39-1 Kurokami, Chuo-ku, Kumamoto 860-8555, Japan
}

\section{A R T I C L E I N F O}

\section{Article history:}

Received 14 July 2015

Received in revised form

9 December 2015

Accepted 15 December 2015

Available online 23 December 2015

\section{Keywords:}

Microwave-assisted hydrothermal

extraction

Malic acid

Kinetic modelling

Synthesis

Low transition temperature mixture

Delignification

\begin{abstract}
A B S T R A C T
This work describes the utilization of microwave hydrothermal extracted malic acid from cactus, lophatherum herb, papaya and Luffa cylindrica as hydrogen bond donor for the synthesis of natural low transition temperature mixtures (LTTMs) with sucrose as hydrogen bond acceptor. The effects of parameters including reaction temperature, extraction time, mass of sample and oxidant, $\mathrm{H}_{2} \mathrm{O}_{2}$ were discussed. The saturation equation was used to fit with the experimental data, observing only a slight loss of the goodness of fit. The optimum conditions for microwave hydrothermal extraction were found to be at $1 \mathrm{~g}$ of sample, $200{ }^{\circ} \mathrm{C}$ and $45 \mathrm{~min}$ in the presence of hydrogen peroxide $\left(\mathrm{H}_{2} \mathrm{O}_{2}\right)$ as oxidant. Consequently, the proposed method was successfully applied to extract malic acid from plants and fruits for the synthesis of LTTMs. The LTTMs prepared from the extracted malic acid have identical physicochemical properties with the LTTMs derived from commercial malic acid due to the existence of hydrogen bond and the ability to dissolve lignin.
\end{abstract}

๑) 2015 Elsevier Ltd. All rights reserved.

\section{Introduction}

Conventional extraction techniques such as soaking, maceration, water percolation and solvent extraction for natural products normally require long extraction time and large volume of organic solvents. These also result into degradation of active compounds obtaining relatively lower extraction efficiency of the target compounds (Moltó-Puigmartí et al., 2011; Chan et al., 2011; Wu et al., 2012). Hydrothermal extraction using water is a natural and green alternative for natural product extraction at high temperature and pressure condition. This method has been used to extract polar organic compounds or to decompose lignocellulosic materials to produce valuable compounds such as saccharides and aromatic acids (Kitada et al., 2009; Matsunaga et al., 2013).

\footnotetext{
* Corresponding author. Tel.: +8196 342 3665; fax: +8196 3423679.

E-mail addresses: ychungloong2009@gmail.com (C.L. Yiin), drsuzana_yusuf@ petronas.com.my (S. Yusup), quitain@kumamoto-u.ac.jp (A.T. Quitain), msasaki@ kumamoto-u.ac.jp (M. Sasaki), yoshimitsu_uemura@petronas.com.my (Y. Uemura), tetsuya@kumamoto-u.ac.jp (T. Kida).
}

Microwave possesses many advantages for the extraction of bioactive compounds (Sanchez-Aldana et al., 2013) such as reduced processing time, higher extraction rates and more accurate control of reaction conditions (Tsubaki et al., 2012; Yemis and Mazza, 2012). Microwave-assisted extraction has been proven to be a promising technique for the extraction of phytochemicals from botanical plants and food samples (Xiao et al., 2012; Quitain et al., 2013). Direct interaction of microwaves helps to heat up the solvent and also lead to a tremendous increase in internal pressure, rupture of plant tissue and release of active compounds into the solvent (Dhobi et al., 2009; Mandal et al., 2007).

Francisco et al., 2012 introduced 26 types of natural low transition temperature mixtures (LTTMs) from environmentally benign starting materials which show glass transition points instead of eutectic melting points. LTTMs can be prepared from cheap, readily available and toxicologically well characterized starting materials (Francisco et al., 2013). Preparation of LTTMs is in vicinity to the synthesis of deep eutectic solvents (Cvjetko Bubalo et al., 2014). The hydrogen bonding interactions of LTTMs lead to some of the promising characteristics of ionic liquids as solvents and shared by deep eutectic solvents (Francisco et al., 2012). The potentials of LTTMs to degrade the lignocellulosic biomass structure were 